\title{
Does immunosuppressive property of non-steroidal anti- inflammatory drugs (NSAIDs) reduce COVID-19 vaccine-induced systemic side effects?
}

\author{
Itsuro Kazama*, Momono Senzaki \\ School of Nursing, Miyagi University, Taiwa-cho, Miyagi, Japan.
}

\begin{abstract}
SUMMARY To help stop the coronavirus disease 2019 (COVID-19) pandemic, vaccines are currently the most critical tool. However, the COVID-19 mRNA vaccines frequently cause systemic side effects shortly after the injection, such as fever, headache and generalized fatigue. In our survey, after receiving the second dose of the COVID-19 vaccine, $80 \%$ developed fever, $62 \%$ headache and $69 \%$ generalized fatigue. Among people who required antipyretics, the average durations of fever and headache were significantly shorter in those who took non-steroidal anti-inflammatory drugs (NSAIDs), such as aspirin, loxoprofen and ibuprofen, than those who took acetaminophen. In our patch-clamp studies, NSAIDs effectively suppressed the delayed rectifier $\mathrm{K}^{+}$-channel (Kv1.3) currents in T-lymphocytes and thus exerted immunosuppressive effects. Because of this pharmacological property, the use of NSAIDs should be more effective in reducing the vaccine-induced systemic side effects that are caused primarily by the enhanced cellular immunity.
\end{abstract}

Keywords Coronavirus disease 2019 (COVID-19), vaccine, side effects, non-steroidal anti-inflammatory drugs (NSAIDs)

Coronavirus disease 2019 (COVID-19), caused by severe acute respiratory syndrome coronavirus 2 (SARS-CoV-2), is still raging worldwide (1). In the absence of drugs or other therapeutics for treating COVID-19, vaccines against the virus are currently the most critical tool to help stop the pandemic (2). Despite the spread of new SARS-CoV-2 variants that are highly contagious, the vaccination of two doses is considered to be effective in reducing the severity of the disease and decreasing the mortality rates (3). However, the administration of COVID-19 mRNA vaccines frequently causes systemic side effects shortly after the injection, such as fever, headache, generalized fatigue, chill, arthralgia, muscle pain and nausea (4). Although they are usually self-limited, serious side effects can cause long-term health problems (5).

In our survey, among 231 Japanese aged 18 to 22 years who received Pfizer/BioNTech vaccines, 23\% developed fever, $25 \%$ headache and $33 \%$ generalized fatigue after the first dose. These percentages markedly increased after the second dose, in which $80 \%$ developed fever, $62 \%$ headache and $69 \%$ generalized fatigue. The findings were consistent with those obtained from recent studies describing that the vaccine-induced side effects after the second dose were more intense than those after the first dose, especially in younger people (4). In our survey, in most people, the symptoms after the first dose subsided spontaneously within 2 days. However, after the second dose, nearly half of the people that developed fever, headache and generalized fatigue required antipyretics, such as acetaminophen and the other nonsteroidal anti-inflammatory drugs (NSAIDs; aspirin, loxoprofen or ibuprofen). The average duration of these symptoms was not significantly different between people who did not take any medications and those who took acetaminophen. However, as shown in Figure 1, the average duration of fever and headache was significantly shorter in those who took NSAIDs than in those who took acetaminophen (Figure $1 \mathrm{~A}$ and $\mathrm{B}$ ).

In addition to producing viral spike proteins, COVID-19 mRNA vaccines stimulate the activity of T-lymphocytes and facilitate their cytokine production (6). Therefore, the systemic side effects caused by the vaccine are thought to be attributable to the enhanced cellular immunity (7). NSAIDs are commonly used as anti-inflammatory, analgesic and antipyretic agents in daily clinical practice. Additionally, due to their immunosuppressive property (8), NSAIDs have also been used in the treatment of autoimmune disorders, including systemic lupus erythematosus and rheumatoid 


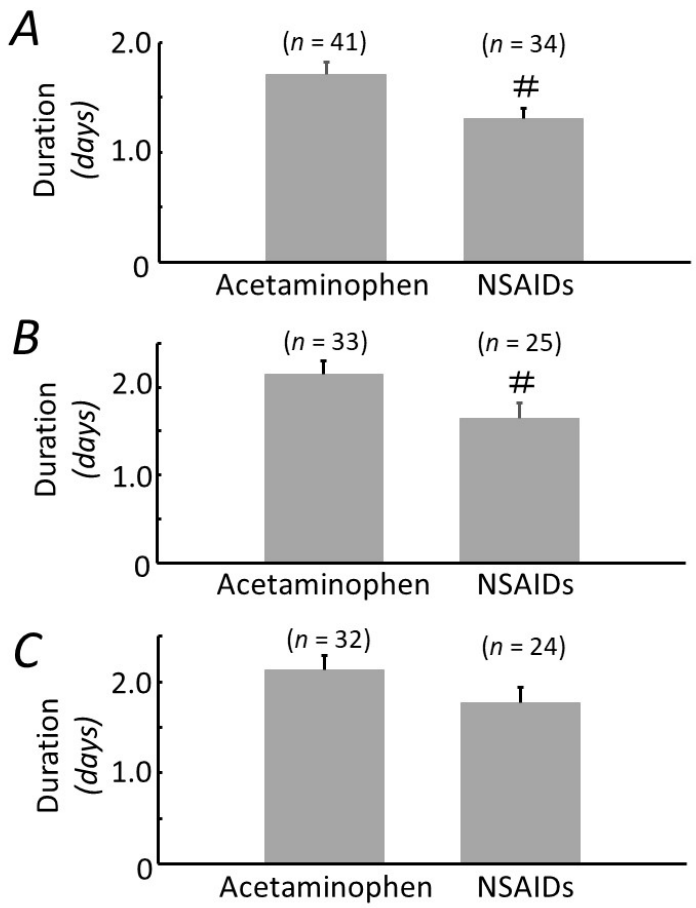

Figure 1. Effects of acetaminophen and non-steroidal antiinflammatory drugs (NSAIDs) on the duration of systemic side effects after receiving the second dose of the COVID-19 vaccines. Durations of fever (A), headache (B) and generalized fatigue (C) after receiving the second dose of the COVID-19 vaccine were compared between those who took acetaminophen and NSAIDs (aspirin, loxoprofen or ibuprofen). ${ }^{*} p<0.05 v s$. those who took acetaminophen. Values are means \pm SEM. Differences were analyzed by ANOVA followed by Dunnett's $t$ test

arthritis (9). In our previous case reports, NSAIDs were actually effective in reducing systemic symptoms triggered by the enhanced autoimmunity $(10,11)$. Concerning the mechanisms by which NSAIDs exert this immunosuppressive property, NSAIDs inhibit the migration of leukocytes or directly suppress their cytokine production, either cyclooxygenase (COX) -dependently or -independently $(12,13)$. Since the enhanced cellular immunity was the primary pathogenesis of the vaccine-induced side effects, the immunosuppressive effect of NSAIDs was thought to be responsible for the more rapid reduction of fever, headache and generalized fatigue in our survey (Figure 1).

In our patch-clamp studies, we have revealed that NSAIDs, such as aspirin, indomethacin and diclofenac, functionally inhibited delayed rectifier $\mathrm{K}^{+}$-channels (Kv1.3) expressed in T-lymphocytes, and thus suppressed the activity of the cells (14) (Figure 2). Since the channels are highly expressed in T-lymphocytes (15), and since selective blockade of the channels actually repressed the immune response in lymphocytes (16), this mechanism was thought to be largely responsible for the immunosuppressive property of NSAIDs (Figure 2). Concerning this pharmacological property, besides the use of immunosuppressive drugs or corticosteroids, the use of selective Kv1.3-

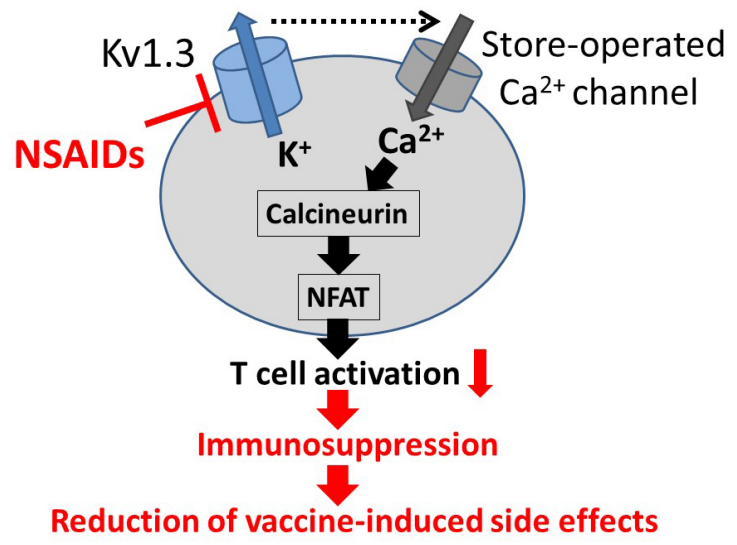

Figure 2. Mechanisms by which non-steroidal anti-inflammatory drugs (NSAIDs) reduce COVID-19 vaccine-induced systemic side effects. Kv1.3-channels promote calcium influx and trigger the proliferation and activation of T-lymphocytes (15). The increased cytosolic calcium concentration stimulates the phosphatase calcineurin, which de-phosphorylates the nuclear factor of activated T cells (NFAT), causing its accumulation in the nucleus and binding to the promoter region of cytokine-encoding genes. NSAIDs inhibit Kv1.3-channels and suppress the activity of T-lymphocytes. Consequently, the drugs exert an immunosuppressive property and reduce the vaccine-induced systemic side effects.

channel inhibitors may also be beneficial. The early administration of these drugs may not only shorten the duration of the vaccine-induced systemic side effects, but also prevent serious complications after the vaccination, such as myocarditis and pericarditis (17). Recently, we have additionally demonstrated in our patch-clamp studies that drugs such as statins (lovastatin, simvastatin), antibiotics (clarithromycin, chloroquine), anti-hypertensive drugs (nifedipine, benidipine, diltiazem, verapamil) and anti-allergic drugs (cetirizine, fexofenadine, azelastine, terfenadine), also strongly suppress the Kv1.3-channel currents in T-lymphocytes $(15,18-20)$. In this context, besides NSAIDs, these drugs may also be potentially effective in reducing vaccine-induced systemic side effects.

Funding: This work was supported by the Salt Science Research Foundation, No. 2123 to IK.

Conflict of Interest: The authors have no conflicts of interest to disclose.

\section{References}

1. Kazama I. Targeting lymphocyte Kv1.3-channels to suppress cytokine storm in severe COVID-19: Can it be a novel therapeutic strategy? Drug Discov Ther. 2020; 14:143-144.

2. Dagan N, Barda N, Kepten E, Miron O, Perchik S, Katz MA, Hernan MA, Lipsitch M, Reis B, Balicer RD. BNT162b2 mRNA Covid-19 vaccine in a nationwide mass vaccination setting. N Engl J Med. 2021; 384:14121423.

3. Lopez Bernal J, Andrews N, Gower C, et al. 
Effectiveness of Covid-19 vaccines against the B.1.617.2 (Delta) variant. N Engl J Med. 2021; 385:585-594.

4. Menni C, Klaser K, May A, et al. Vaccine side-effects and SARS-CoV-2 infection after vaccination in users of the COVID Symptom Study app in the UK: a prospective observational study. Lancet Infect Dis. 2021; 21:939-949.

5. Kazama I. Stabilizing mast cells by commonly used drugs: a novel therapeutic target to relieve post-COVID syndrome? Drug Discov Ther. 2020; 14:259-261.

6. Teijaro JR, Farber DL. COVID-19 vaccines: modes of immune activation and future challenges. Nat Rev Immunol. 2021; 21:195-197.

7. Woldemeskel BA, Garliss CC, Blankson JN. SARSCoV-2 mRNA vaccines induce broad CD4+ T cell responses that recognize SARS-CoV-2 variants and HCoV-NL63. J Clin Invest. 2021; 131.

8. Cho JY. Immunomodulatory effect of nonsteroidal antiinflammatory drugs (NSAIDs) at the clinically available doses. Arch Pharm Res. 2007; 30:64-74.

9. Wallace DJ. Advances in drug therapy for systemic lupus erythematosus. BMC Med. 2010; 8:77.

10. Kazama I, Sasagawa N, Nakajima T. Complete remission of human parvovirus b19 associated symptoms by loxoprofen in patients with atopic predispositions. Case Rep Med. 2012; 2012:703281.

11. Kazama I, Miura C, Nakajima T. Nonsteroidal antiinflammatory drugs quickly resolve symptoms associated with EBV-induced infectious mononucleosis in patients with atopic predispositions. Am J Case Rep. 2016; 17:8488 .

12. Hackstein H, Morelli AE, Larregina AT, Ganster RW, Papworth GD, Logar AJ, Watkins SC, Falo LD, Thomson AW. Aspirin inhibits in vitro maturation and in vivo immunostimulatory function of murine myeloid dendritic cells. J Immunol. 2001; 166:7053-7062.

13. Gao JX, Issekutz AC. The effect of ebselen on polymorphonuclear leukocyte migration to joints in rats with adjuvant arthritis. Int J Immunopharmacol. 1993; 15:793-802.

14. Kazama I, Maruyama Y, Murata Y. Suppressive effects of nonsteroidal anti-inflammatory drugs diclofenac sodium, salicylate and indomethacin on delayed rectifier $\mathrm{K}^{+}$channel currents in murine thymocytes. Immunopharmacol Immunotoxicol. 2012; 34:874-878.

15. Kazama I. Physiological significance of delayed rectifier $\mathrm{K}^{+}$channels (Kv1.3) expressed in $\mathrm{T}$ lymphocytes and their pathological significance in chronic kidney disease. $\mathrm{J}$ Physiol Sci. 2015; 65:25-35.

16. Villalonga N, David M, Bielanska J, Gonzalez T, Parra D, Soler C, Comes N, Valenzuela C, Felipe A. Immunomodulatory effects of diclofenac in leukocytes through the targeting of Kv1.3 voltage-dependent potassium channels. Biochem Pharmacol. 2010; 80:858866.

17. Diaz GA, Parsons GT, Gering SK, Meier AR, Hutchinson IV, Robicsek A. Myocarditis and pericarditis after vaccination for COVID-19. JAMA. 2021; 326:1210-1212.

18. Kazama I, Baba A, Maruyama Y. HMG-CoA reductase inhibitors pravastatin, lovastatin and simvastatin suppress delayed rectifier $\mathrm{K}^{+}$-channel currents in murine thymocytes. Pharmacol Rep. 2014; 66:712-717.

19. Baba A, Tachi M, Maruyama Y, Kazama I. Suppressive effects of diltiazem and verapamil on delayed rectifier $\mathrm{K}^{+}$channel currents in murine thymocytes. Pharmacol Rep. 2015; 67:959-964.

20. Saito K, Abe N, Toyama H, Ejima Y, Yamauchi M, Mushiake H, Kazama I. Second-generation histamine H1 receptor antagonists suppress delayed rectifier $\mathrm{K}^{+}$-channel currents in murine thymocytes. Biomed Res Int. 2019; 2019:6261951.

Received October 12, 2021; Revised October 17, 2021; Accepted October 24, 2021.

*Address correspondence to:

Itsuro Kazama, School of Nursing, Miyagi University, 1-1 Gakuen, Taiwa-cho, Kurokawa-gun, Miyagi 981-3298, Japan. E-mail:kazamai@myu.ac.jp

Released online in J-STAGE as advance publication October 26, 2021. 